Article

\title{
Hybrid Microencapsulated Phase-Change Material and Carbon Nanotube Suspensions toward Solar Energy Conversion and Storage
}

\author{
Jun $\mathrm{Li}^{1}$, Lisi Jia ${ }^{2, *}$, Longjian Li ${ }^{1}$, Zehang Huang ${ }^{2}$ and Ying Chen ${ }^{2}$ \\ 1 School of Energy and Power Engineering, Chongqing University, Chongqing 400044, China; \\ lijuncqu@foxmail.com (J.L.); longjian@cqu.edu.cn (L.L.) \\ 2 School of Materials and Energy, Guangdong University of Technology, Guangzhou 510006, China; \\ gduthzh@foxmail.com (Z.H.); chenying@gdut.edu.cn (Y.C.) \\ * Correspondence: jialisi@gdut.edu.cn
}

Received: 28 July 2020; Accepted: 25 August 2020; Published: 26 August 2020

\begin{abstract}
In this study, a new type of functional hybrid suspension for solar energy conversion and thermal energy storage was prepared by adding carbon nanotube (CNT) and microencapsulated phase-change material (MEPCM) into deionized water. MEPCM with octadecane as the core material and titania $\left(\mathrm{TiO}_{2}\right)$ as the shell material was synthesized by the sol-gel method. The MEPCMs were spherical particles with diameters of 2-4 $\mu \mathrm{m}$, and the thickness of the shell was about $100 \mathrm{~nm}$. The MEPCM achieved better thermal stability and thermal conductivity than the pure octadecane due to the $\mathrm{TiO}_{2}$ shell. The melting and solidification latent heats of the MEPCM were about 154.24 and $154.26 \mathrm{~J} / \mathrm{g}$, respectively. The encapsulation efficiency of octadecane was calculated to be $65.84 \%$. Most of all, the novel hybrid CNT and MEPCM suspensions exhibited remarkable dispersion stability owing to the stable reticular structure composed of $\mathrm{CNT}$ in the suspension. Compared with pure water, the thermal conductivity, specific heat of the MEPCM/CNT suspension improved by 34.48 and $43.57 \%$, respectively and the photo-thermal conversion efficiency reached a high value of $86.0 \%$. This work provided a new type of hybrid functional suspension towards direct absorption solar collector for solar energy conversion and storage.
\end{abstract}

Keywords: microencapsulated phase-change material; carbon nanotube; direct absorption solar collector; heat storage; photo-thermal conversion efficiency

\section{Introduction}

At present, environmental pollution and energy shortage have become two major global problems to be solved urgently. The development of clean and sustainable energy has attracted huge attention in recent years. As an inexhaustible new energy source, solar energy has great significance in alleviating the energy crisis due to its wide sources and its properties of being clean and pollution-free [1-3]. Solar photo-thermal conversion technology is one of the most important solar energy technologies, which can convert solar radiation energy into heat energy by reflecting or absorbing sunlight. However, the traditional solar thermal conversion systems, such as solar thermal collector, are limited by time, space, and weather. Direct absorption solar collector (DASC) is a new technology that uses some medium with a large specific heat to directly absorb the solar radiation and convert the energy to heat to store. DASC is an effective way to solve the mismatch between solar energy supply and demand, as well as improve the efficiency of photo-thermal conversion.

Water, ethylene glycol, and thermal oil, which are low-cost and have large specific heat, are widely used in the solar collector system for thermal energy storage and heat transfer. However, they have some disadvantages such as low absorbance and thermal conductivity. To improve the photo-thermal 
conversion efficiency and thermal conductivity, different kinds of nanoparticle were added into the liquid to prepare the two-phase slurries. Qu et al. [4] prepared nanofluid by adding multi-wall carbon nanotubes and $\mathrm{CuO}$ nanoparticles into water and carried out an experimental study on its photo-thermal conversion efficiency. The results showed that CuO-MWCNT nanoparticles could enhance the photo-thermal conversion efficiency of suspension, the maximum terminal temperature rise of $14.1^{\circ} \mathrm{C}$ was achieved concerning water. He et al. [5] studied the photo-thermal conversion efficiency of $\mathrm{Cu}-\mathrm{H}_{2} \mathrm{O}$ nanofluid in the solar band, and the results showed that when the mass concentration of $\mathrm{Cu}$ was $0.1 \%$, the maximum temperature that nanofluid could reach was $25.3 \%$ higher than that of pure water. Sani et al. [6] prepared glycol nanofluids using single-walled carbon nanohorn, and the results showed that single-walled carbon nanohorn played a significant role in improving the solar absorption characteristics of nanofluids. Zeiny et al. [7] verified the photo-thermal conversion characteristics of nanofluids prepared from gold, copper and carbon black respectively through experiments, and the results showed that the nanoparticles could improve the photo-thermal conversion efficiency of base fluids, and carbon black showed the most significant improvement, indicating that carbon-based materials had better photo-thermal conversion performance. Gorji et al. [8] prepared water-based nanofluids using hydrophilic modified carbon nanotubes, and the results showed that carbon nanotubes could significantly improve the absorption of sunlight and photo-thermal conversion efficiency of the water. In summary, adding nanoparticles to the base solution can significantly improve the photo-thermal conversion performance. However, due to the low specific heat of the nanoparticles, the heat storage capacities of the slurries are reduced, which greatly limits the solar energy storage.

In recent decades, many studies have shown that adding appropriate phase-change microcapsules into the base solution can improve the specific heat capacity of the base solution. Phase-change microcapsule is a new type of energy storage material with a core-shell structure, it can store and release a large amount of thermal energy during the phase transition process. In recent years, some researchers tried to fabricate MEPCMs with special shell materials, which may also improve the photo-thermal conversion capacity. Chen et al. [9] prepared carbon nanotubes as the shell material, and studied the photo-thermal conversion performance of the MEPCM suspension, and found that the photo-thermal conversion performance and thermal conductivity of the suspension were significantly improved compared with pure water. Gao et al. [10] coated eicosane with cuprous oxide and prepared the MEPCM into water. The results showed that the specific heat capacity, thermal conductivity, and light-heat conversion capacity of the suspension were also greatly improved. These new kinds of MEPCM took into account the performance of thermal energy storage and photo-thermal conversion. However, it can be also found that the shell materials of these phase-change microcapsules were limited, and the preparation method was complex, these microcapsules were not yet suitable for large-scale practical applications. Presently, most of the microcapsules were fabricated by polymer, silicon dioxide $\left(\mathrm{SiO}_{2}\right)$, and titanium dioxide $\left(\mathrm{TiO}_{2}\right)$ [11-13]. However, there are still many shortcomings of the MEPCMs, such as low heat charge and discharge rate, poor photo-thermal conversion efficiency, short life span, poor chemical stability, etc. How to apply these MEPCMs to DASC is still a challenge.

Some studies have shown that the hybrid suspension composed of nanoparticles and phase-change microcapsules can combine their respective advantages and improve the thermal conductivity and specific heat capacity of the base solution at the same time [14-16]. However, the studies of hybrid nanoparticles and phase-change microcapsule suspension were mainly about the slurry rheological property and energy storage capacity. There is still a lack of research on hybrid nanoparticle and MEPCM suspension for DASC. In the current work, a heat storage suspension containing n-octadecane@ $\mathrm{TiO}_{2}$ microcapsules and CNT was prepared to improve the photo-thermal conversion efficiency of the DASC system. $\mathrm{TiO}_{2}$ has been widely used as the shell material of phase-change microcapsule. Octadecane has a huge latent heat and the phase transition temperature ranges from $25-28^{\circ} \mathrm{C}$, which is a comfortable temperature range for human in winter. It is very suitable for the DASC that used for the room temperature regulation. Carbon nanotube (CNT) is a carbon-based material with good absorbance and thermal conductivity. The hybrid suspension was prepared by a two-step method. First, MEPCM in 
which octadecane as the core and $\mathrm{TiO}_{2}$ as the shell was prepared by a sol-gel method. Then a small amount of carbon nanotube and phase-change microcapsule were added into the water to prepare hybrid suspension for further study. The morphology, heat capacity, thermal conductivity, dispersion stability, and photo-thermal conversion efficiency of the MEPCM and hybrid suspension were characterized. The octadecane@ $\mathrm{TiO}_{2}$ microcapsules exhibited high thermal energy storage capability and thermal conductivity. The thermal stability of MEPCM was improved by the $\mathrm{TiO}_{2}$ shell. The hybrid suspension showed outstanding dispersion stability, thermal conductivity, specific heat, and photo-thermal conversion performance. This novel hybrid CNT-MEPCM suspension can be a potential candidate in DASC for solar energy conversion and storage.

\section{Materials and Methods}

\subsection{Materials}

n-Octadecane (AR), sodium dodecyl sulfate (SDS, AR) and carboxylic multi-walled carbon nanotubes (CNT, AR) were purchased from Shanghai Aladdin Reagent Co., Ltd. (Shanghai, China) Tetrabutyl titanate (TBT, AR) and formamide (AR) were purchased from Shanghai Macklin Reagent Co., Ltd. (Shanghai, China). The deionized water was produced by a pure water system (Zhiang clever series, Shanghai, China).

\subsection{Synthesis of Octadecane@TiO $\mathrm{O}_{2} \mathrm{MEPCM}$}

The synthesis process of the octadecane@ $\mathrm{TiO}_{2} \mathrm{MEPCM}$ is illustrated in Figure 1. The typical procedure involved three steps: First, $5 \mathrm{~g}$ of octadecane and $1 \mathrm{~g}$ of SDS were added to $100 \mathrm{~mL}$ of formamide and well mixed with a magnetic stirrer at $50{ }^{\circ} \mathrm{C}$ for $30 \mathrm{~min}$, which resulted in an oil-water emulsion. Then $5 \mathrm{~mL}$ of TBT was added to the emulsion dropwise under continuous stirring for $30 \mathrm{~min}$. Finally, $2.5 \mathrm{~g}$ deionized water was mixed with $20 \mathrm{~mL}$ formamide and then slowly dripped into the emulsion to start the sol-gel reaction of TBT. After reaction for $5 \mathrm{~h}$, the MEPCM particles were separated from the solution using a centrifuge. Then the particles were washed with water and ethanol 3 times to remove the remaining reactants. After freeze-drying for $8 \mathrm{~h}$, the MEPCMs were completely dry.

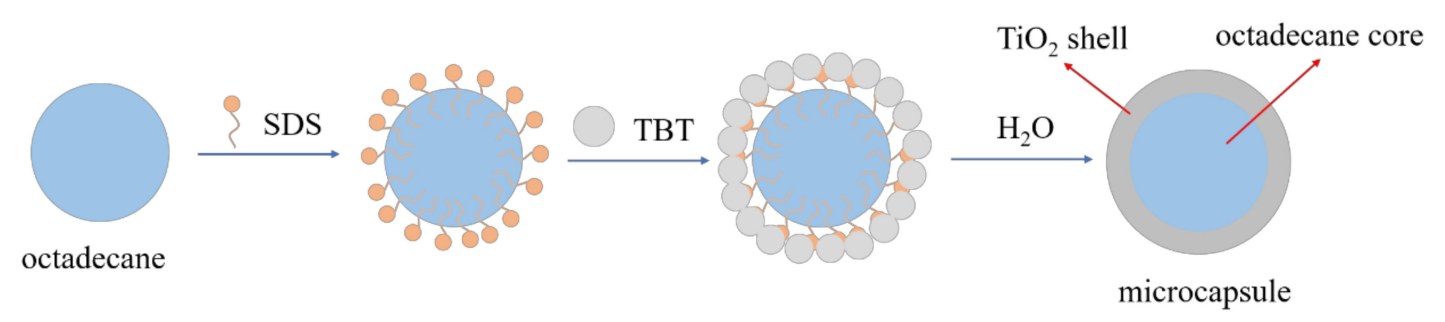

Figure 1. Schematic illustration of the synthesis process of n-octadecane@ $\mathrm{TiO}_{2}$ microcapsules.

\subsection{Characterization and Measurement}

The morphology and chemical compositions of the n-octadecane@ $\mathrm{TiO}_{2}$ microcapsules and CNT were investigated by field-emission scanning electron microscopy (FESEM, S-4800, Hitachi) and transmission electron microscopy (TEM, JEM-2100, JEOL). The specimens were coated with a thin layer of gold-palladium alloy which made them electrically conductive. The composition analysis of the n-octadecane, $\mathrm{TiO}_{2}$ shell, and MEPCMs were performed using Fourier transform infrared spectroscopy (FT-IR, Nicolet 6700). The thermal properties of the MEPCMs were characterized by a differential scanning calorimeter (DSC, DSC-3, Mettler Toledo, $\pm 1 \%$ ). The thermal stability of the MEPCMs was investigated by the thermal gravimetric analyzer (TGA, SDT 2960, $\pm 2 \%$ ) with a heating rate of $10^{\circ} \mathrm{C} / \mathrm{min}$ from 50 to $500^{\circ} \mathrm{C}$ under nitrogen. The thermal conductivities of the MEPCM and MEPCM/CNT suspension were measured by the transient plane source method using a thermal conductivity meter (TPS 500S, Hot Disk, $\pm 2 \%$ ). 


\subsection{Photo-Thermal Conversion Performance of MEPCM/CNT Suspension}

To identify the photo-thermal conversion performance of MEPCM/CNT suspension, a simulated solar irradiation system was used, which was presented in Figure 2. The experiment was carried as follows. The same mass of the MEPCM and different mass of CNT were put into a glass beaker, then a settled water was added in the beaker. After $1 \mathrm{~h}$ of ultrasonic dispersion, the hybrid MEPCM/CNT suspension got stable. The thermal conductivity and specific heat of the suspensions were measured by thermal conductivity meter and DSC. The temperature change of the sample during the experiment were recorded by an Agilent $34970 \mathrm{~A}+$ Agilent $34901 \mathrm{~A}$ temperature collection recorder $\left( \pm 0.5^{\circ} \mathrm{C}\right)$. The light to thermal conversion and storage efficiency $(\eta)$ was calculated using the equation below.

$$
\eta=\frac{m \int C_{p}(T) d T}{G_{s} A t} \times 100 \%
$$

where $m$ is the mass of the suspension, $C_{p}(T)$ is the specific heat of the suspension, $T$ is the temperature of the suspension, $G_{S}$ is the irradiance of the light, $A$ is the surface area of the suspension, $t$ is the time.

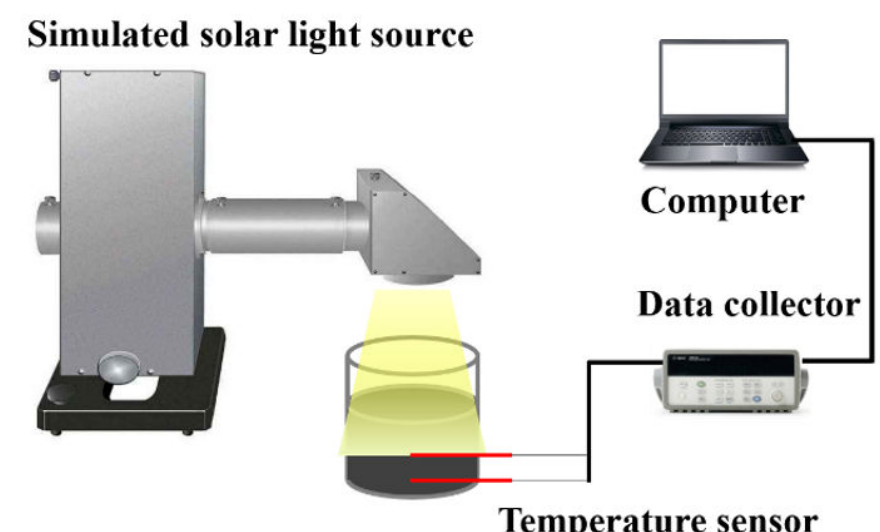

Figure 2. The experimental apparatus for evaluating the photo-thermal conversion performance.

\section{Results and Discussion}

\subsection{Morphology and Chemical Structure of MEPCMs}

As is shown in Figure 3, the morphology and microstructure of the MEPCM containing an octadecane core and $\mathrm{TiO}_{2}$ shell was observed using the FESEM. In Figure 3a, it was clear to see that all the microcapsules had a uniform spherical structure. The surface of the microcapsules was also very smooth and compact. This was mostly because the capsules were synthesized under a moderate reaction condition. The water used in the reaction was limited, which may offer better control of the reaction process. Figure $3 \mathrm{~b}$ showed a dissected microcapsule, the capsule had a distinct core-shell structure, which can be well proved the successful encapsulation of octadecane into the $\mathrm{TiO}_{2}$ shell. The thickness of the shell was about $100 \mathrm{~nm}( \pm 2 \mathrm{~nm})$, which can hold the octadecane inside and help to overcome the leakages and cracking. The diameter of the microcapsules was measured by Dynamic light scattering (DLS, DelsaNano C, Backman Counter), and the results are shown in Figure 3c. It revealed that the average particle size of the prepared MEPCMs was about $2900 \mathrm{~nm}$.

Figure $3 \mathrm{~d}$ showed the FT-IR curves of the MEPCM, $\mathrm{TiO}_{2}$ and n-octadecane. For n-octadecane, there were two strong peaks at $2920 \mathrm{~cm}^{-1}$ and $2850 \mathrm{~cm}^{-1}$ which were because of the stretching of the $\mathrm{C}-\mathrm{H}$ bond of methylene deformation vibration [17]. The peaks at $1469 \mathrm{~cm}^{-1}$ and $1384 \mathrm{~cm}^{-1}$ were ascribed to the deformation vibration of $-\mathrm{CH}_{2}$ and $-\mathrm{CH}_{3}$, while the peak at $718 \mathrm{~cm}^{-1}$ belonged to the in-plane rocking vibration of $-\mathrm{CH}_{2}$ [18]. The wide peaks around $3400 \mathrm{~cm}^{-1}$ and $1600 \mathrm{~cm}^{-1}$ on the $\mathrm{TiO}_{2}$ curve were correspond to the stretch vibration of the $\mathrm{O}-\mathrm{H}$ bond, which may be located on the surface 
of the particles or caused by water in the sample [19]. For the shell and MEPCM IR curves, the IR transmittance was weak at wavenumber below $1000 \mathrm{~cm}^{-1}$, which may prove the existence of Ti-O-Ti group [20]. As shown in Figure 3d, the FT-IR spectrum of MEPCM was nearly the superposition of the n-octadecane and the $\mathrm{TiO}_{2}$ shell spectra. The results of FT-IR indicated that the MEPCM which had an n-octadecane core and $\mathrm{TiO}_{2}$ shell had been fabricated, successfully.
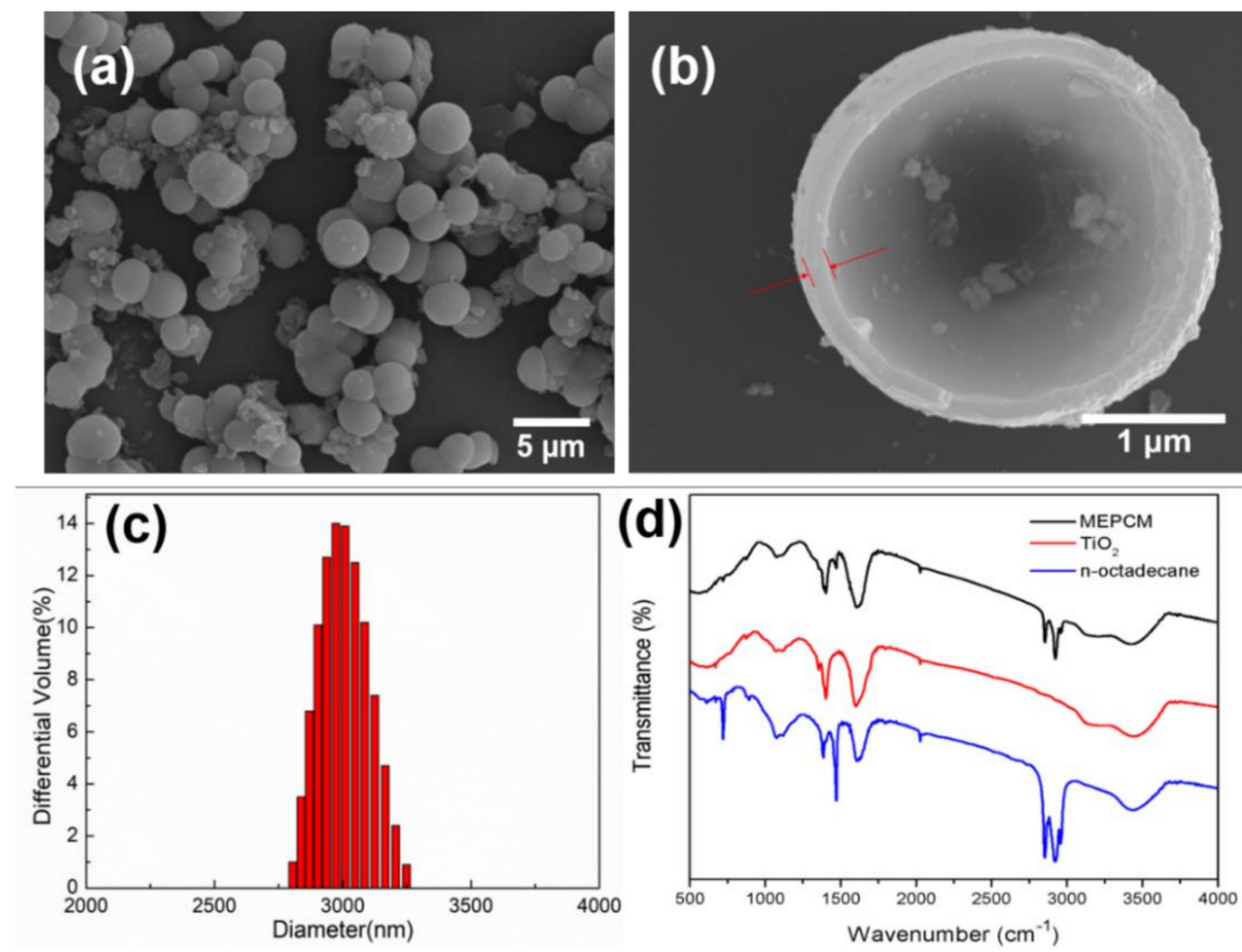

Figure 3. Characterizations of phase-change microcapsule: (a) FESEM image of MEPCMs; (b) Microstructure of the microcapsule; (c) Size distribution of the MEPCMs; (d) FT-IR spectra of the MEPCMs.

\subsection{Thermal Properties of the MEPCMs}

The latent heat was an important thermophysical property for the MEPCMs when they were used for thermal energy storage. The phase-change temperature and latent heat of octadecane and MEPCM were measured by the DSC. Figure $4 a$, b were the DSC curves of the solidifying and melting process of the MEPCMs, and the thermophysical properties of the samples are listed in Table 1. The pure n-octadecane had high phase-change enthalpies over $229.18 \pm 1.02 \mathrm{~J} / \mathrm{g}$, which indicated it could be an ideal thermal energy storage material. However, the phase-change enthalpy of the MEPCMs was about $154.26 \pm 1.11 \mathrm{~J} / \mathrm{g}$. The MEPCMs consisted of the $\mathrm{PCM}$ core and $\mathrm{TiO}_{2}$ shell, and the $\mathrm{TiO}_{2}$ shell of the MEPCM did not show any latent heat and its sensible heat was also very little, so the phase-change enthalpy of the MEPCMs was contributed by the core materials only. Therefore, the latent heat of phase-change capsules is lower than that of pure phase-change materials, and the greater the proportion of shell material in capsule species, the smaller the latent heat of MEPCM. Usually, encapsulation ratio $\left(E_{e n}\right)$ was used to characterize the proportion of core material in MEPCM, and it can be calculated from the melting enthalpy in Table 1 by Equation (2), and the $E_{\text {en }}$ of the MEPCMs was about $65.84 \%$.

$$
E_{e n}=\frac{\Delta H_{m, \text { micro }}}{\Delta H_{m, o c t a}} \times 100 \%
$$

where $\Delta H_{m, \text { micro }}$ and $\Delta H_{m, o c t a}$ are the melting enthalpy of the MEPCMs and n-octadecane, respectively. All the paraments calculated by Equation (2) were also shown in Table 1. 

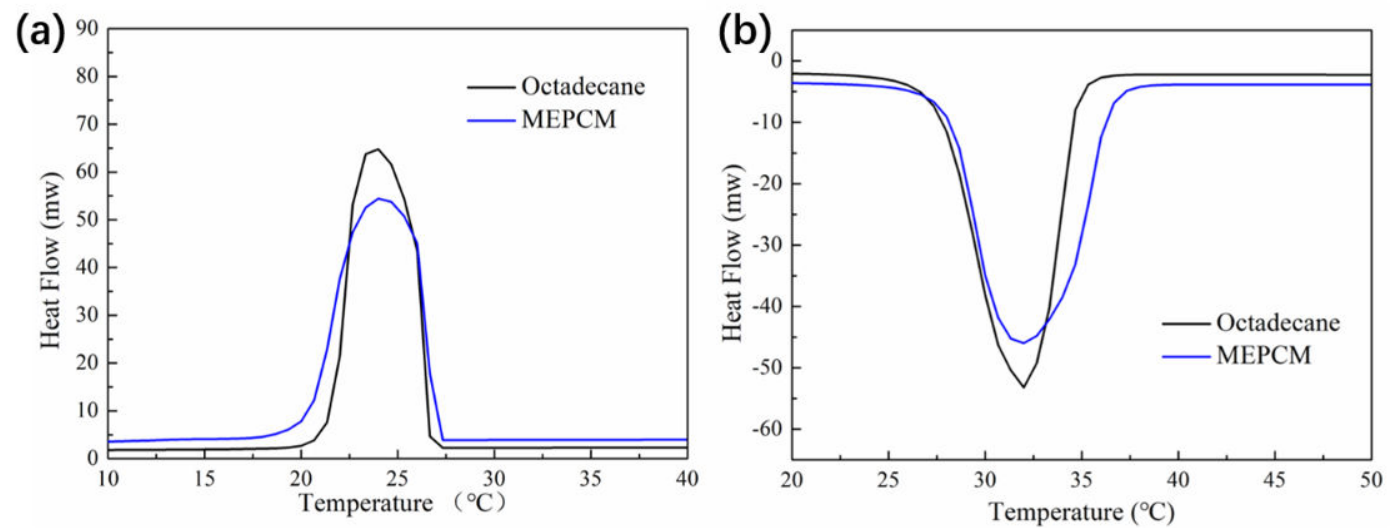

Figure 4. DSC curves of the MEPCMs and octadecane: (a) solidifying and (b) melting.

Thermal conductivity was an important evaluation index for the MEPCMs, as it may affect the heat transfer rate and thermal response. The thermal conductivities of the pure n-octadecane and MEPCMs are shown in Table 1 . The thermal conductivity of the n-octadecane was $0.16 \pm 0.03 \mathrm{~W} \mathrm{~m}^{-1}$ $\mathrm{K}^{-1}$; however, the thermal conductivity of MEPCMs was about $0.55 \pm 0.02 \mathrm{~W} \mathrm{~m}^{-1} \mathrm{~K}^{-1}$. It was obvious to see that the thermal conductivity of the MEPCMs was enhanced markedly 3 times compared with the pure n-octadecane, indicating that the $\mathrm{TiO}_{2}$ shell increased the conductivity of the capsules.

Table 1. Thermal characteristics of the n-octadecane and MEPCM.

\begin{tabular}{|c|c|c|c|c|c|}
\hline \multirow[b]{2}{*}{ Sample } & \multicolumn{2}{|c|}{ Melting } & \multicolumn{2}{|c|}{ Solidifying } & \multirow{2}{*}{$\begin{array}{c}\text { Thermal } \\
\text { Conductivity } \\
\left(\mathrm{W} \mathrm{m}^{-1} \mathrm{~K}^{-1}\right)\end{array}$} \\
\hline & $\begin{array}{c}\text { Peak } \\
\text { Temperature } \\
\left({ }^{\circ} \mathrm{C}\right)\end{array}$ & $\begin{array}{c}\text { Latent Heat } \\
(\mathrm{J} / \mathrm{g})\end{array}$ & $\begin{array}{c}\text { Peak } \\
\text { Temperature } \\
\left({ }^{\circ} \mathrm{C}\right)\end{array}$ & $\begin{array}{c}\text { Latent Heat } \\
(\mathrm{J} / \mathrm{g})\end{array}$ & \\
\hline N-octadecane & 32.71 & $234.26 \pm 0.94$ & 23.67 & $229.18 \pm 1.02$ & $0.16 \pm 0.03$ \\
\hline МЕРСM & 33.41 & $154.24 \pm 1.09$ & 24.82 & $154.26 \pm 1.11$ & $0.55 \pm 0.02$ \\
\hline
\end{tabular}

\subsection{Thermal Stability of $M E P C M$}

The thermal stability of the capsules was an important parament of the MEPCMs when they were used for thermal energy storage. Figure 5 showed the TGA curves of the MEPCM and pure octadecane. The temperature with $5 \%$ weight loss was defined as the onset decomposition temperature $\left(\mathrm{T}_{\text {onset }}\right)$, which was used to evaluate the thermal stability of the material. The $\mathrm{T}_{\text {onset }}$ of the octadecane was about $122.37^{\circ} \mathrm{C}$. The $\mathrm{T}_{\text {onset }}$ of the MEPCM was about $136.02{ }^{\circ} \mathrm{C}$, which was higher than that of octadecane. It was obvious that the $\mathrm{TiO}_{2}$ shell improved the thermal stability of the MEPCMs.

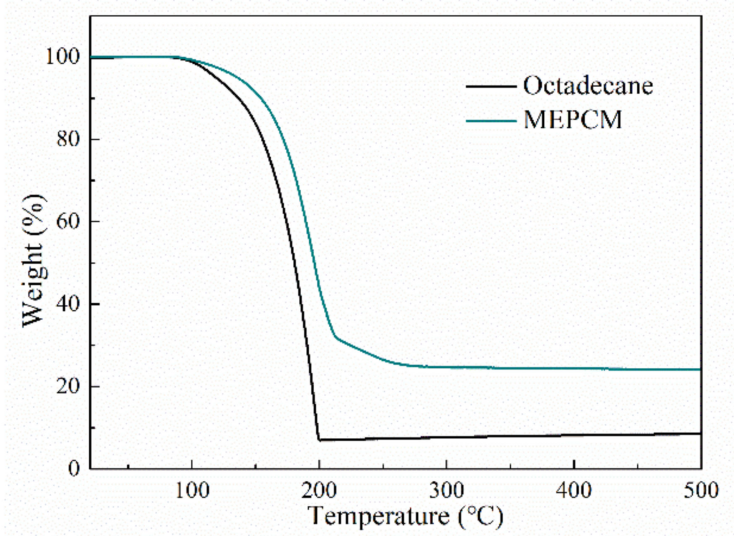

Figure 5. TGA curves of n-octadecane and MEPCM. 


\subsection{Dispersion Stability of MEPCM/CNT Suspension}

The hybrid suspension was prepared by dispersing the MEPCM and CNT into water. Figure 6 shown the images of the MEPCM and MEPCM/CNT suspensions at different standing times. The color of the hybrid suspensions did not change over time; however, the MEPCM suspension changed into transparent after 7 days for the MEPCM deposited at the bottom by gravity. To explain the phenomenon, the structure of the CNT in the suspension was investigated using the TEM. A typical TEM image of the CTN is shown in Figure 7a. The CNTs were not dispersed in the water uniformly, on the contrary, many CNTs were interwoven with each other. It seemed almost inevitable that the MEPCMs were encircled by stable CNT nets and restricted in space. Hence, the aggregation and sedimentation process of the capsules was greatly slowed. Figure $7 \mathrm{~b}$ was diagrammatic of this microstructure in the hybrid suspension. The hybrid MEPCM/CNT suspension had good dispersion stability, which will facilitate the high stability and efficiency of its thermal performance.

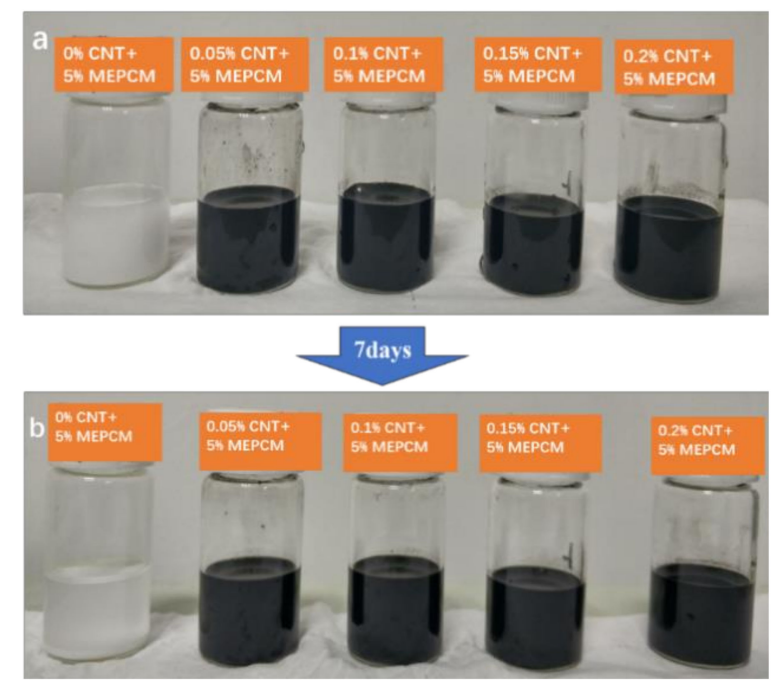

Figure 6. Typical sedimentation photographs of the suspension: (a) 0 day and (b) 7 days.

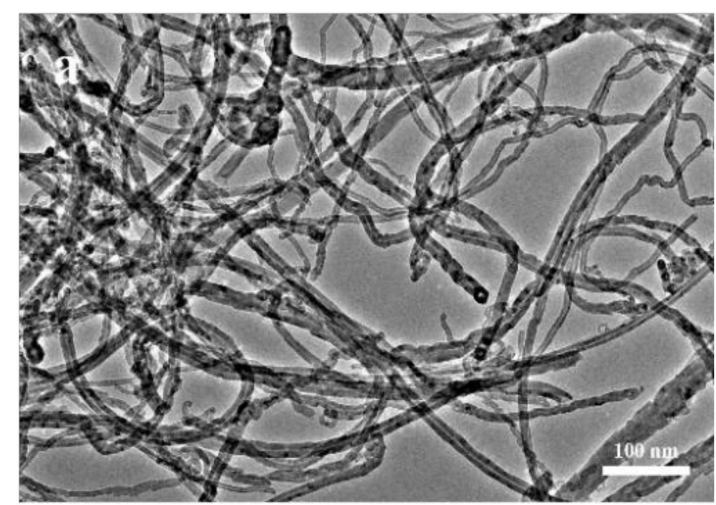

(a)

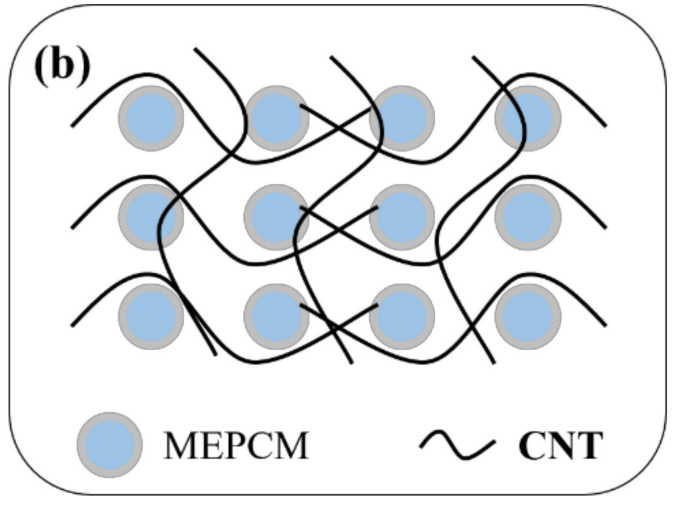

(b)

Figure 7. (a)TEM photograph of the CTN; (b) Schematic illustration of the hybrid suspension.

\subsection{Thermal-Physical Properties of Hybrid MEPCM/CNT Suspension}

Figure 8 showed the thermal conductivity curves of water and hybrid suspension with different CNT concentrations varying with temperature. The thermal conductivities of MEPCM/CNT suspension and pure water gradually increased with the increase of temperature. This was because the rise in temperature will intensify the natural convection of the fluid and the microscopic movement of the particles, thus enhancing the effect of heat conduction. Compared with pure water, the thermal 
conductivity of MEPCM suspension decreased slightly. However, Adding CNT can significantly improve the thermal conductivity of MEPCM suspension. As shown in Figure 8, the thermal conductivity of MEPCM/CNT mixed suspension was higher than that of pure water, and the improvement degree became more obvious with the increase of CNT concentration. For example, when the mass concentration of CNT was $0.2 \mathrm{wt} . \%$, the thermal conductivity of the MEPCM/CNT mixed suspension increased from 0.78 to $0.83 \mathrm{~W} /(\mathrm{m} \cdot \mathrm{K})$ with the increase of temperature, which was about 34.48 and $29.69 \%$ higher than that of the pure water, and about 41.82 and $36.07 \%$ higher than that of the MEPCM suspension, respectively. The higher thermal conductivity was conducive to the faster transfer of heat in the working medium and improved the heat storage and release rate of the working medium in the process of photo-thermal energy storage.

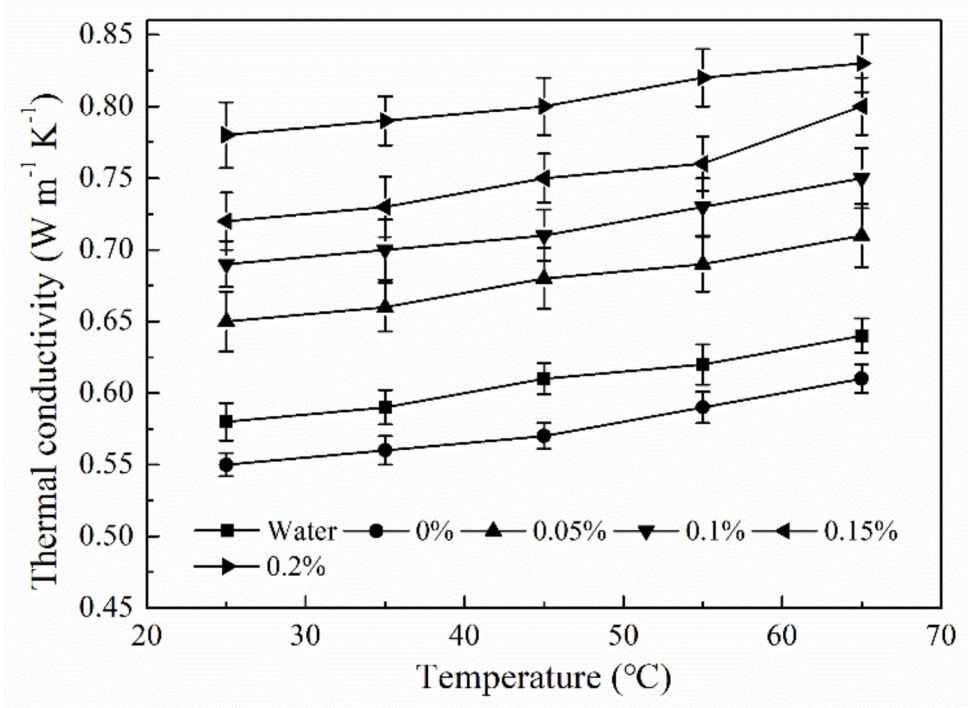

Figure 8. Thermal conductivity curves of water and hybrid suspension with different CNT concentrations varying with temperature.

\subsection{Specific Heat of Hybrid MEPCM/CNT Suspension}

Figure 9 showed the specific heat capacity curves of MEPCM/CNT suspension with different $\mathrm{CNT}$ concentrations as a function of temperature. It can be seen from the figure that within the phase transition temperature range of MEPCM particles $\left(25-35^{\circ} \mathrm{C}\right)$, the specific heat capacity of mixed suspension was significantly increased. This was because MEPCM particles undergo a melting phase transition in this temperature range and absorb a large amount of heat, which increased the specific heat capacity of the suspension. The MEPCM suspension had the largest $C_{P}$ of $6.57 \pm 0.03 \mathrm{~J} /(\mathrm{g} \cdot \mathrm{K})$. When the mass concentration of CNT increased from 0 to $0.2 \%$, the $C_{P}$ of suspension decreased from $6.57 \mathrm{~J} /(\mathrm{g} \cdot \mathrm{K})$ to $6.03 \pm 0.03 \mathrm{~J} /(\mathrm{g} \cdot \mathrm{K})$. This is because CNT has no latent heat, and the sensible heat of CNT is very small, so adding $C N T$ will decrease the $C_{P}$ of suspension. However, the $C_{P}$ of hybrid suspension was still much higher than the pure water $\left(C_{P}=4.20 \mathrm{~J} /(\mathrm{g} \cdot \mathrm{K})\right)$ at $30^{\circ} \mathrm{C}$ [9]. Therefore, in the phase-change temperature range of the MEPCM, the specific heat of the mixed suspension had a greater improvement than that of the base solution, and the larger specific heat capacity is more conducive to improving the energy storage and heat transfer capacity of the working medium.

\subsection{Photo-Thermal Performance of Hybrid MEPCM/CNT Suspension}

To investigate the photo-thermal conversion process, the MEPCM suspension and MEPCM/CNT suspensions were heated by a solar simulator, the light intensity from the light was about $1 \pm 0.03 \mathrm{~W} / \mathrm{cm}^{2}$. The temperatures of the suspensions were recorded by the thermocouple and a data collector. The results are shown in Figure 10. The temperatures of the pure water and MEPCM suspension were slowly increased from room temperature to $31.7 \pm 0.8^{\circ} \mathrm{C}$ and $49.0 \pm 1.1^{\circ} \mathrm{C}$, respectively. The pure water was 
transparent, so most of the light passed through the liquid, rather than be absorbed. The light reflected by the MEPCM suspension with a milky white surface and little light energy can be converted into heat energy, these were the main reasons for the results. The temperature of the MEPCM/CNT suspensions presented quickly rise with the increase of irradiation times, and the maximal temperatures were much higher than pure water and MEPCM suspension. The temperature of the hybrid suspension showed a slight increase with the increase of CNT concentration. This is due to the CNT has good absorbance and photo-thermal conversion efficiency. Meanwhile, CNT also improves the thermal conductivity of suspension, so it can effectively improve the photo-thermal conversion efficiency of mixed suspension. During the light on the process, the temperature increase of the MEPCM/CNT suspensions presented a hysteresis at $25-28{ }^{\circ} \mathrm{C}$, indicating the MEPCMs started the fusion. The light to thermal energy conversion efficiency $(\eta)$ for the MEPCM/CNT suspensions was calculated based on Equation (1). The photo-thermal conversion efficiencies of the MEPCM/CNT suspensions were about $79.5 \pm 1.3 \%, 80.8 \pm 1.5 \%, 81.7 \pm 1.9 \%, 86.0 \pm 1.5 \%$ when the CNT content ranging from $0.05,0.1$, 0.15 and $0.2 \%$. The light to thermal energy conversion efficiency increased slightly with the increase of CNT concentration, indicating that just a little CNT can hugely improve the light to the thermal energy conversion efficiency of the hybrid suspension.

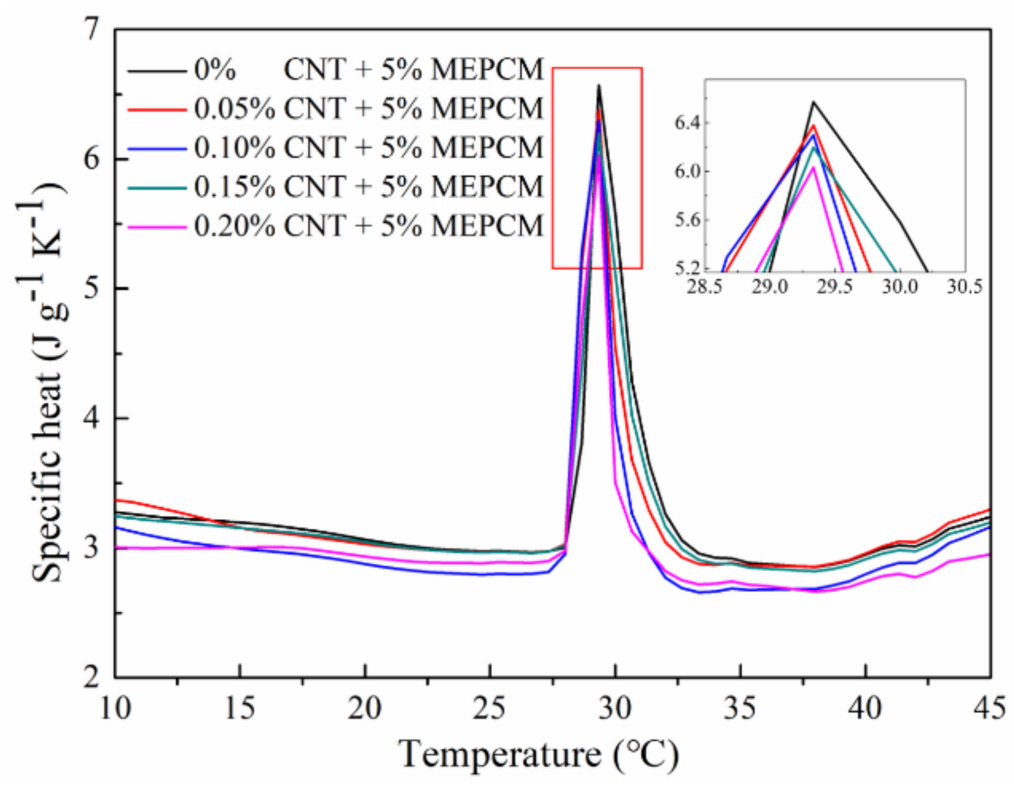

Figure 9. Specific heat capacity curves of MEPCM/CNT suspension with different CNT concentrations.

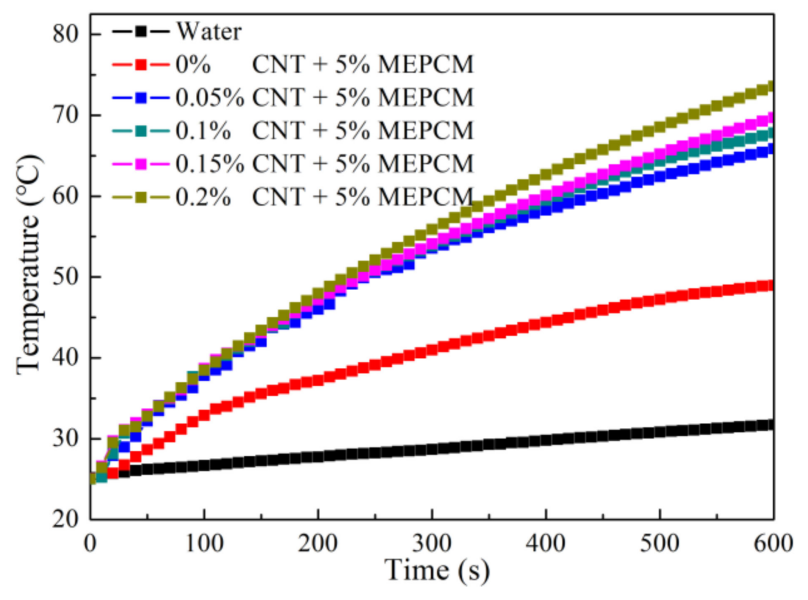

Figure 10. Temperature variation curves with irradiation time of water and hybrid suspensions. 


\section{Conclusions}

In summary, we reported a simple method of increasing the photo-thermal conversion and storage of water for the DASC by preparing a hybrid MEPCM/CNT suspension. The thermophysical properties and photo-thermal conversion performance were studied, and the main conclusions were drawn as follows:

1. The n-octadecane@ $\mathrm{TiO}_{2}$ composite microcapsules were successfully synthesized through an in situ hydrolysis and polycondensation reaction in a nonaqueous emulsion. The morphology and thermal properties of the MEPCMs were characterized. The SEM results showed that the prepared microcapsules displayed a uniform spherical shape and a well-defined core-shell structure with a smooth surface, the thickness of the shell was about $100 \mathrm{~nm}$. The average diameter of the MEPCMs was about $2.9 \mu \mathrm{m}$ and had a narrow distribution, which was confirmed by the DLS test. The FT-IR results confirmed the successful encapsulation of n-octadecane into $\mathrm{TiO}_{2}$ shell. The DSC measurements indicated that the MEPCMs had a large latent heat of $154 \mathrm{~J} / \mathrm{g}$ and the encapsulated ratio was about $65.84 \%$. The TGA results showed that the $\mathrm{TiO}_{2}$ shell of the MEPCMs improved thermal stability.

2. Due to the net structure of CNT in the water, the dispersion stability of the MEPCM/CNT suspension was higher than that of MEPCM suspension. The thermal conductivity of the MEPCM/CNT suspension can reach $0.83 \mathrm{~W} /(\mathrm{m} \cdot \mathrm{K})$, which was about $29.7 \%$ higher than that of the pure water. The specific heats of the MEPCM/CNT suspension were higher than water due to the phase change of the MEPCM. The photo-thermal conversion efficiency of MEPCM/CNT suspension reaches $86.0 \%$ because the hybrid suspension exhibited higher thermal conductivity and optical absorption property than the pure water. It can be concluded that the hybrid MEPCM/CNT suspension with enhanced thermophysical and photo-thermal conversion performance paves the way for the application of the binary system of MEPCM and nanoparticles towards improving the effective receiver efficiency of DASC.

Author Contributions: Investigation, Y.C.; Formal analysis, L.J.; Data curation, Z.H.; Writing-review \& editing L.J., L.L.; Writing—original draft, J.L. All authors have read and agreed to the published version of the manuscript.

Funding: This research was funded by the National Nature Science Foundation of China grant number 51876045 and 51606045, and the Department of Science and Technology of Guangdong Province [2017TX04N371].

Conflicts of Interest: The authors declare no conflict of interest.

\section{References}

1. Xu, B.; Xu, J.; Chen, Z. Heat transfer study in solar collector with energy storage. Int. J. Heat Mass Transf. 2020, 156, 119778. [CrossRef]

2. Timilsina, G.R.; Kurdgelashvili, L.; Narbel, P.A. A review of solar energy: Markets, economics and policies. Policy Res. Work. Pap. 2011, 16, 1.

3. Huang, Z.F.; Pan, L.; Zou, J.J.; Zhang, X.; Wang, L. Nanostructured bismuth vanadate-based materials for solar-energy-driven water oxidation: A review on recent progress. Nanoscale 2014, 6, 14044-14063. [CrossRef] [PubMed]

4. Qu, J.; Zhang, R.; Wang, Z.; Wang, Q. Photo-thermal conversion properties of hybrid CuO-MWCNT/H2O nanofluids for direct solar thermal energy harvest. Appl. Therm. Eng. 2019, 147, 390-398. [CrossRef]

5. He, Q.; Wang, S.; Zeng, S.; Zheng, Z. Experimental investigation on photothermal properties of nanofluids for direct absorption solar thermal energy systems. Energy Convers. Manag. 2013, 73, 150-157. [CrossRef]

6. Sani, E.; Mercatelli, L.; Barison, S.; Pagura, C.; Agresti, F.; Colla, L.; Sansoni, P. Potential of carbon nanohorn-based suspensions for solar thermal collectors. Sol. Energy Mater. Sol. Cells 2011, 95, $2994-3000$. [CrossRef]

7. Zeiny, A.; Jin, H.; Bai, L.; Lin, G.; Wen, D. A comparative study of direct absorption nano fluids for solar thermal applications. Sol. Energy 2018, 161, 74-82. [CrossRef] 
8. Gorji, T.B.; Ranjbar, A.A.; Mirzababaei, S.N. Optical properties of carboxyl functionalized carbon nanotube aqueous nanofluids as direct solar thermal energy absorbers. Sol. Energy 2015, 119, 332-342. [CrossRef]

9. Chen, Y.; Zhang, Q.; Wen, X.; Yin, H.; Liu, J. A novel CNT encapsulated phase change material with enhanced thermal conductivity and photo-thermal conversion performance. Sol. Energy Mater. Sol. Cells 2018, 184, 82-90. [CrossRef]

10. Gao, F.; Wang, X.; Wu, D. Design and fabrication of bifunctional microcapsules for solar thermal energy storage and solar photocatalysis by encapsulating para ffi $\mathrm{n}$ phase change material into cuprous oxide. Sol. Energy Mater. Sol. Cells 2017, 168, 146-164. [CrossRef]

11. Jamekhorshid, A.; Sadrameli, S.M.; Farid, M. A review of microencapsulation methods of phase change materials (PCMs) as a thermal energy storage (TES) medium. Renew. Sustain. Energy Rev. 2014, 31, 531-542. [CrossRef]

12. Zhao, C.Y.; Zhang, G.H. Review on microencapsulated phase change materials (MEPCMs): Fabrication, characterization and applications. Renew. Sustain. Energy Rev. 2011, 15, 3813-3832. [CrossRef]

13. Giro-Paloma, J.; Martínez, M.; Cabeza, L.F.; Fernández, A.I. Types, methods, techniques, and applications for microencapsulated phase change materials (MPCM): A review. Renew. Sustain. Energy Rev. 2016, 53, 1059-1075. [CrossRef]

14. Zhang, G.H.; Zhao, C.Y. Thermal property investigation of aqueous suspensions of microencapsulated phase change material and carbon nanotubes as a novel heat transfer fluid. Renew. Energy 2013, 60, 433-438. [CrossRef]

15. Ho, C.J.; Huang, J.B.; Tsai, P.S.; Yang, Y.M. Preparation and properties of hybrid water-based suspension of $\mathrm{Al} 2 \mathrm{O} 3$ nanoparticles and MEPCM particles as functional forced convection fluid. Int. Commun. Heat Mass Transf. 2010, 37, 490-494. [CrossRef]

16. Jia, L.; Li, Y.; Chen, Y.; Wang, J.; Mo, S.; Li, J.; Liu, G. New hybrid suspension of MEPCM/GO particles with enhanced dispersion stability and thermo-physical properties. Appl. Energy 2019, 255, 113827. [CrossRef]

17. Yu, S.; Wang, X.; Wu, D. Microencapsulation of n-octadecane phase change material with calcium carbonate shell for enhancement of thermal conductivity and serving durability: Synthesis, microstructure, and performance evaluation. Appl. Energy 2014, 114, 632-643. [CrossRef]

18. Li, H.; Fang, G.; Liu, X. Synthesis of shape-stabilized paraffin/silicon dioxide composites as phase change material for thermal energy storage. J. Mater. Sci. 2010, 45, 1672-1676. [CrossRef]

19. Connor, P.A.; Dobson, K.D.; James McQuillan, A. Infrared spectroscopy of the $\mathrm{TiO}_{2} /$ aqueous solution interface. Langmuir 1999, 15, 2402-2408. [CrossRef]

20. Zimbone, M.; Cacciato, G.; Buccheri, M.A.; Sanz, R.; Piluso, N.; Reitano, R.; La Via, F.; Grimaldi, M.G.; Privitera, V. Photocatalytical activity of amorphous hydrogenated $\mathrm{TiO}_{2}$ obtained by pulsed laser ablation in liquid. Mater. Sci. Semicond. Process. 2016, 42, 28-31. [CrossRef] 